\title{
Identification of Histone Epigenetic Modifications with Chromatin Immunoprecipitation PCR Array in Chronic Lymphocytic Leukemia Specimens
}

\section{Gwen Jordaan, Wei Liao, Natalie Coriaty and Sanjai Sharma*}

UCLA Greater Los Angeles Veterans Affairs Healthcare System and Division of Hematology Oncology UCLA West Los Angeles VA Medical Center, Los Angeles, California, USA

\begin{abstract}
Histone epigenetic modifications are one of the frequently observed epigenetic alterations in leukemic cells. To efficiently identify histone acetylation status of a number of gene promoters in chronic lymphocytic leukemia (CLL) specimens, we performed a combination of chromatin immunoprecipitation (ChIP) and PCR array. ChIP by an antipan acetylated $\mathrm{H} 4$ histone antibody was followed by a PCR amplification of gene promoters $(n=84)$ to determine their histone acetylation status. A subset of genes with differential histone acetylation status was identified in CLL specimens, and their RNA expression level was correlated with real time quantitative RT-PCR analysis (qRT-PCR). Globally CLL specimens have more hypo-acetylated or epigenetically silenced gene promoters as compared to normal peripheral-blood mononuclear cells. Promoters of E-cadherin, Fos, CDKN2B, c-Jun, BAX genes were found to be histone hypo-acetylated while the promoters of cyclin D1, CDK4, Myc, and TGF beta were hyper-acetylated. The histone acetylation status correlated well with qRT-PCR analysis of a number of CLL specimens. Based on the ChIP-PCR array epigenetically silenced genes and transcriptionaly active genes can be identified. Histone deacetylase inhibitors (HDACi) induce apoptosis in CLL specimens and to determine changes in gene transcription induced by HDACi the ChIP-PCR array was performed with HDACi treatment. Interestingly the HDACi exposure resulted in histone hyper-acetylation of a subset of genes with increased expression of CDKN2B, c-Jun and BAX RNA while promoters of pro-growth and survival genes such as BCL-2, NFKB1, beta-catenin and CDK4 underwent promoter histone hypo-acetylation and downregulation of their RNA expression. The ChIP-PCR array assay can be utilized to detect histone modifications in the genome of leukemic cells and reflects the transcriptional status of the genes.
\end{abstract}

Keywords: CLL; Histones; Epigenetics; Histone deacetylase inhibitors; Chromatin immunoprecipitation

\section{Introduction}

Epigenetic modifications such as DNA methylation and histone modifications alter the transcriptional state of a number of genes and are involved in leukemia initiation and progression [1-3]. These modifications are heritable, reversible and alter expression patterns of a number of genes without altering any DNA sequences. Chronic lymphocytic leukemia (CLL) is a common leukemia in the elderly population that is characteristically associated with a number of genetic and epigenetic alterations. In this leukemia, genetic alterations such as chromosomal deletions involving chromosome 11q, 13q, 17p along with DNA amplifications such as trisomy 12 have clinical and prognostic significance [4]. Besides genetic alterations, a number of genes in CLL are reportedly silenced by epigenetic modifications [5-7]. There is a growing list of genes that are epigenetically dysregulated in CLL specimens, e.g. wnt pathway gene SFRP1 [8,9], Zap-70 [10,11], DAPK1 [12] and more recently micro RNA expression was found to be modulated by epigenetic changes [13].

Genes are epigenetically altered by mechanisms that include DNA cytosine methylation by DNA methyl transferases and modifications such as methylation, phosphorylation and acetylation of the histones [1-3]. In addition to packaging the DNA; the histones also regulate chromatin dynamics and gene expression [14]. Acetylation is a post translation modification that is induced by a set of competing histone modifying enzymes that increase or decrease the histone acetylation [15]. Increase in histone acetylation reflects an increase in gene transcription while epigenetic silencing of genes results in histone hypo-acetylation. Inhibitors of this DNA epigenetic modification are recognized as promising anticancer agents that allow re-expression of silenced genes, cell cycle arrest and apoptosis [15-17]. Histone deacetylase inhibitors (HDACi) inhibit the histone deacetylases (HDACs) and lead to a change in overall compactness of the chromatin promoting accessibility of the DNA to the transcription factors and gene transcription [15]. The methodology for identification of epigenetic alterations in the genome has evolved with development of techniques that rapidly identify a large number of epigenetic alterations in leukemia cells [5-7,18-20]. The experimental approaches have included genome wide studies with microarray analysis and recently high-throughput whole genome sequencing in combination with chromatin immunoprecipitation. A drawback of these approaches is that data analysis is cumbersome and requires a high level of expertise that is not readily available. In this study, we have analyzed the histone epigenetic alterations of gene promoters in CLL specimens by a PCR array methodology in conjunction with chromatin immunoprecipitation to determine if this technique could be used to efficiently analyze epigenetic alterations in

*Corresponding author: Sanjai Sharma, UCLA West Los Angeles VA Medica Center, 11301 Wilshire Blvd, Bldg 304, Rm E1-115, Los Angeles, CA 90073, USA Tel: 3104783711 ext: 83622; Fax: 3102684508; E-mail: sasharma@mednet.ucla.edu

Received June 23, 2014; Accepted August 28, 2014; Published September 02 , 2014

Citation: Jordaan G, Liao W, Coriaty N, Sharma S (2014) Identification of Histone Epigenetic Modifications with Chromatin Immunoprecipitation PCR Array in Chronic Lymphocytic Leukemia Specimens. J Cancer Sci Ther 6: 325-332. doi:10.4172/1948-5956.1000290

Copyright: (c) 2014 Jordaan G, et al. This is an open-access article distributed under the terms of the Creative Commons Attribution License, which permits unrestricted use, distribution, and reproduction in any medium, provided the original author and source are credited. 
leukemic cells. The array findings were additionally correlated with RT-PCR data from primary CLL specimens.

\section{Material and Methods}

\section{Cell culture and reagents}

Peripheral blood samples were obtained from patients at the Los Angeles VA hospital after informed consent and institutional approval. CLL specimens were isolated by ficoll density gradient and stored in liquid nitrogen. Isolates are patients with more than 90\% CLL cells in the ficoll preparation. Normal peripheral- blood mononuclear cells (PBMC) were obtained from voluntary donors and isolated by the ficoll density gradient method. HDACi MS275 (ChemieTek, Indianapolis, IN) was dissolved in DMSO and used at concentrations described. CLL cells were cultured in RPMI 1640 containing $10 \%$ FCS, 1 mM sodium pyruvate, $2 \mathrm{mM} \mathrm{L}$-glutamine and penicillin-streptomycin solution (ATCC, Manassas, VA).

\section{Chromatin immunoprecipitation}

The ChIP assay was performed according to the protocol of the EZChIP ${ }^{\mathrm{TM}}$ Chromatin Immunoprecipitation kit (Millipore, MA). About 3 $\times 10^{6}$ cells treated with MS275 were fixed with $1 \%$ formaldehyde. The cross-linked histone-DNA complexes were sheared to 200-1000 base pair fragments and immunoprecipitated with a pan acetyl $\mathrm{H} 4$ antibody. The immunoprecipitated DNA was then purified and amplified as described below.

\section{ChIP qPCR array}

Epitect ChIP qPCR Array for human oncogenes and tumor suppressor genes was obtained from Qiagen (Valencia,CA). A 96-well plate containing primers specific for promoter regions of 84 genes was used. A cocktail containing the ChIP DNA samples was prepared as per protocol, and quantitatively analyzed by real-time PCR. For each specimen, two PCR plates were analyzed. One contained total input DNA and the other contained ChIP immunoprecipitated DNA. Fold enrichment by the pan acetyl histone $\mathrm{H} 4$ antibody was calculated using the $\mathrm{Ct}$ values from both the plates. The fold enrichment data from two CLL samples was averaged to determine the acetylation status of the various promoters. The CLL samples were compared with and without MS275 treatment ( $1 \mu \mathrm{M} 24$ hours) to determine the promoters that are acetylated with HDACi exposure.

\section{Real-time reverse transcription PCR analysis}

RNA was isolated as described in the RNAeasy Mini kit by Qiagen. Total RNA from pooled B-cells (CD19+) that had been prepared from normal donors was purchased from Invitrogen (Carlsbad, CA). cDNA was synthesized using oligo-dT, dNTP and SuperScript II Reverse Transcriptase from Invitrogen. Relative expression of selected genes from the PCR array was determined using inventoried probes and PCR master mix by Applied Biosystems (Foster City, CA). Real time PCR expression of actin was used as an endogenous control. All PCR experiments, including ChIP qPCR array were performed on Applied Biosystems StepOnePlus machine and the relative expression calculated as described by Pfaffl [21].

\section{Statistical analysis}

Results are shown as the mean \pm standard deviation (SD) of values obtained in three or more independent experiments. Statistical analysis was performed using the Student's t-test using the GraphPad software.

\section{Results}

\section{Promoter ChIP-PCR analyses}

To assess promoter histone acetylation status of a number of genes in CLL specimens a ChIP-PCR array was performed. The array "Oncogenes and Tumor suppressor array" includes a pre-defined set of 84 important genes that are known to function as cell cycle regulators, oncogenes, tumor suppressor genes, pro and anti-apoptotic genes. Chromatin from two CLL specimens (CLL\#4, \#7) and two normal peripheral-blood mononuclear cells (PBMC) were subjected to immunoprecipitation with the pan acetylated histone $\mathrm{H} 4$ antibody. PBMC specimens were used as a control as it is difficult to obtain a large number of purified B cells for chromatin immunoprecipitation. This antibody only recognizes the acetylated histone $\mathrm{H} 4$ that is present in the genomic DNA fragments associated with actively transcribed DNA in the cell. The total input DNA and the immunoprecipitated DNA was analyzed by two separate real time PCR reactions on the array. Based on the cycle difference between the total input DNA and the acetylated histone H4 DNA a ratio (fold enrichment index) was calculated for all the genes in the normal PBMC and CLL specimens. The data from two normal PBMC cell specimens were averaged, and the raw data sets are in supplementary data 1 .

To compare the acetylation status of the gene promoters in CLL specimens relative to PBMC, the fold enrichment of the CLL specimens was divided by the fold enrichment of the PBMC specimens. This categorizes gene promoters who are hypo or hyper acetylated as compared to normal PBMC specimens (Figure 1). Bar diagram shows data from two CLL specimens $(\# 4, \# 7)$, the gene promoters listed on the right side of the midline are the hyper-acetylated promoters (values above 1) and the gene promoters on the left side of the midline are the hypo-acetylated promoters (values below 1) relative to the PBMC cells. The CLL specimens have a small proportion of hyper-acetylated gene promoters as compared to PBMC. In the two CLL specimens, $34 \%$ and $21 \%$ of analyzed gene promoters are hyper-acetylated and the rests are hypo-acetylated as compared to PBMC. Some examples of genes with hyper-acetylated promoters in the two CLL specimens that are biologically relevant include MYC, CDK4, CCND1, BCL2, and TGFB1 [22-26]. These genes are also known to be actively transcribed in CLL specimens as compared to PBMC indicating that the histone $\mathrm{H} 4$ acetylation status is a surrogate marker for active transcription. A large number of gene promoters in the two CLL specimens are, however, hypo-acetylated or silenced. Some of the hypo-acetylated genes promoters implying repressed or silenced transcription with potential or known function in CLL include, BAX, FOS, CDKN2A, B and $\mathrm{CDH} 1$ [27-31].

\section{Validation of the ChIP array data by RT-PCR}

To validate the data from the ChIP array, a number of genes relevant to CLL biology were selected from this analysis to determine whether there was a correlation between the promoter acetylation status and its RNA expression by real time PCR. In Figure 2, the data from real time PCR analysis of a number of primary CLL specimens is shown relative to the expression in pooled normal peripheral blood B cells ( $n=3$, arbitrary value of 1$)$. BAX [27] expression (Figure 2A) in CLL specimens $(n=11)$ is lower than pooled normal B cells (mean $\pm S D$, $0.44 \pm 0.15,8$ of 10 CLL specimens, $n=10$ ) and is a gene that has a hypoacetylated promoter on the ChIP-PCR array. BCL2 [25] expression is higher in CLL specimens as compared to control B cells (mean $\pm S D$, $2.01 \pm 0.69)$ however; its promoter was hypo-acetylated in one of the CLL specimens (CLL\#4). CDK4 [23] gene promoter is hyper-acetylated 


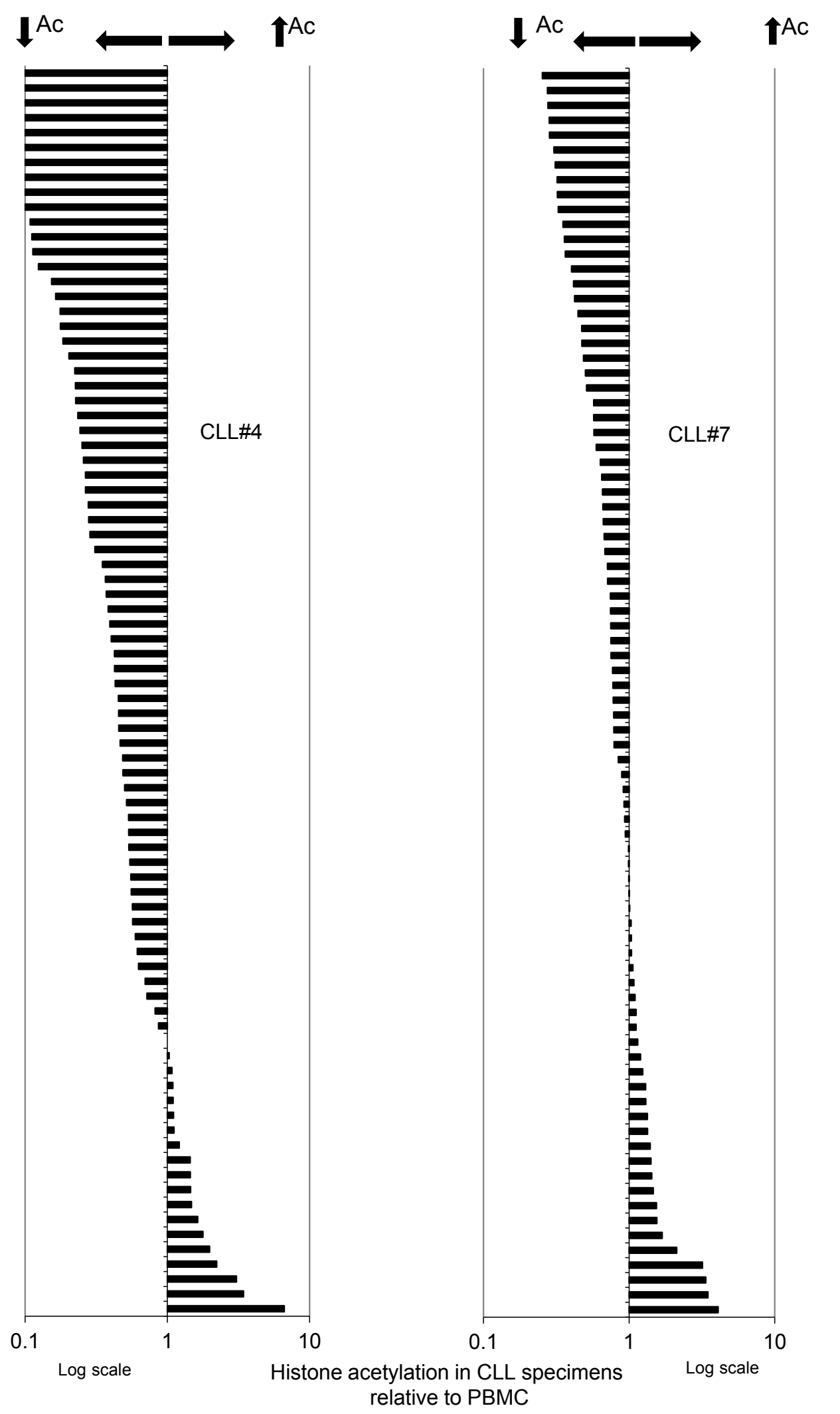

Figure 1: ChIP qPCR data of two CLL specimens with enrichment relative to the normal PBMC. The enrichment index of each gene promoter of CLL specimens was divided by the enrichment index of the normal PBMC. Horizontal axis shows the enrichment index of the promoter of genes on the array with both negative (hypoacetylated relative to PBMC) as well as positive (hyper-acetylated relative to PBMC) values (linear scale). The gene promoters are also listed serially with positive values at the bottom to negative on the top. 
A

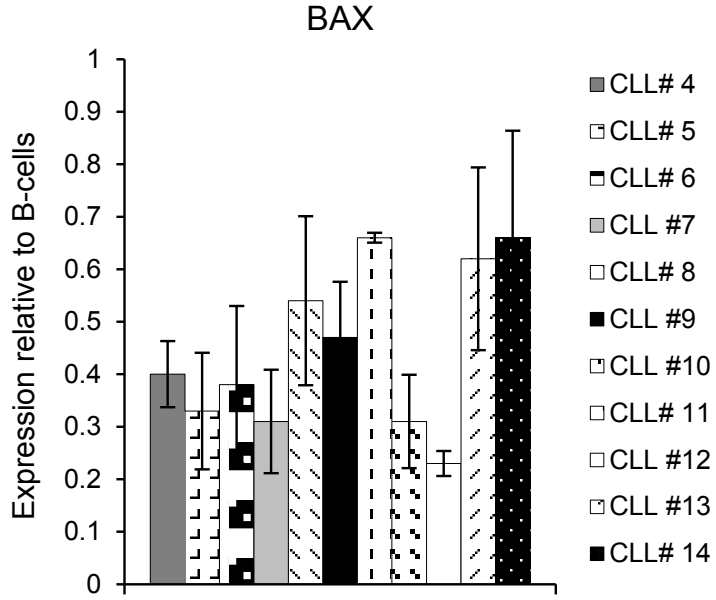

C

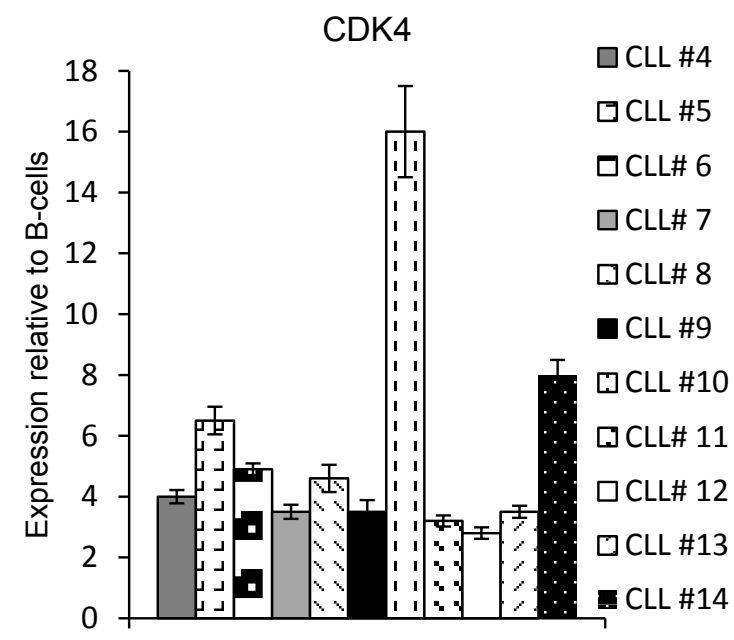

B

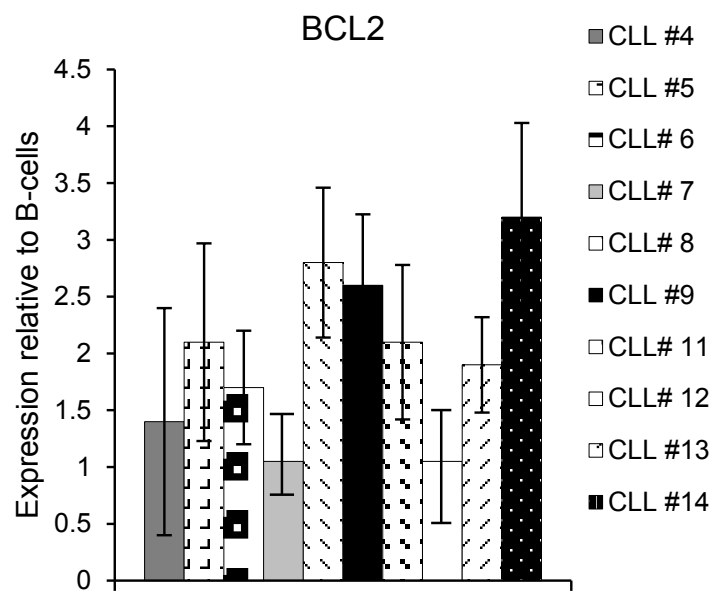

D

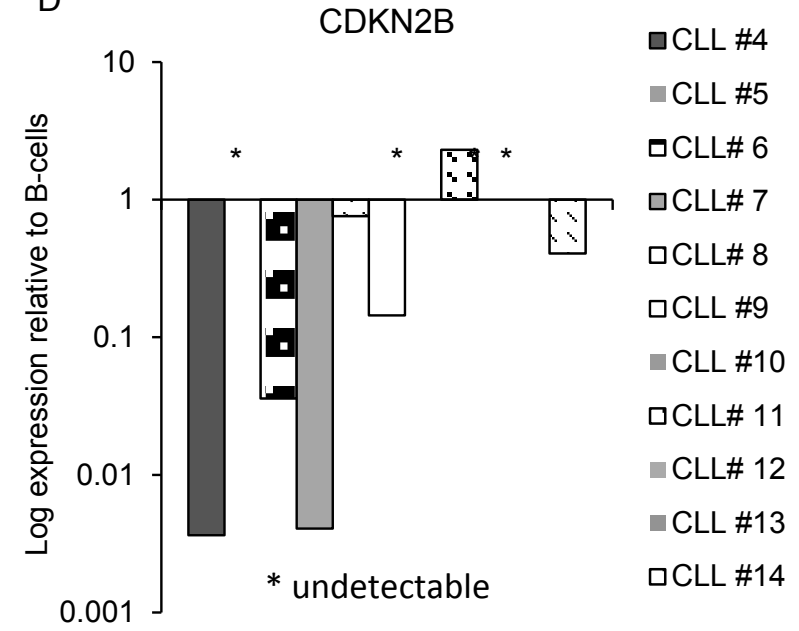

Figure 2: Real time PCR analysis of selected genes in CLL specimens: BAX, BCL2, CDK4, and CDKN2B RNA were analyzed in primary CLL specimens with Taqman probes and real time PCR. All the data is relative to the expression in B cells isolated from normal donor PBMCs ( $n=3$, mean and SD).

in both the CLL specimens (\#4, \#7) and its RNA was over-expressed in all $(\mathrm{n}=11)$ the CLL specimens tested. In the ChIP array data (Figure 2) the CDKN2B [30] promoter is hypo-acetylated in CLL\#4, and was therefore tested in additional CLL specimens. The CDKN2B expression was either undetectable or low in 10 of 11 CLL specimens. Overall, the data shows a good concordance between ChIP PCR array histone acetylation status and RT-PCR analysis for CLL specimens. The acetylation status of the promoters using a pan $\mathrm{H} 4$ acetylation specific antibody is an indirect way of assessing the transcriptional state of a gene promoter.

\section{Promoter ChIP-PCR analysis of HDACi treated CLL specimens}

To determine the alterations in gene expression with HDACi exposure in CLL specimens and to find out if the ChIP-PCR array can be used to study the mechanism of action of HDACi, histone acetylation statuses of promoters were analyzed after HDAC inhibition. A number of HDAC inhibitors such as valproic acid, MGCD0103, SAHA (Vorinostat) and MS275 (Entinostat) are able to induce apoptosis in CLL specimens $[17,24,32,33]$. In preliminary experiments with SAHA, Valproic acid and MS275, the HDAC inhibitor MS275 was most effective in inducing apoptosis in primary CLL specimens at equimolar concentration and was selected for further experiments. CLL\#4, \#7 was treated with MS275 at a concentration of $1 \mu \mathrm{m}$ for 24 hours and analyzed with the ChIP-PCR array [24]. This duration of HDACi exposure was selected as at longer exposure, significant apoptosis was observed CLL specimens [unpublished observations]. Figure 3 shows the data comparing the acetylation status of the promoters in CLL specimen with HDACi MS275 exposure relative to the untreated specimen. This data was calculated by dividing the fold enrichment after HDACi exposure with fold enrichment of the untreated CLL specimen. The bar diagram indicates that the promoter acetylation status of a number of genes is altered with HDACi exposure in CLL specimens.

Genes that increase promoter acetylation with HDACi exposure in the two CLL specimens are on the right side of the bar diagram. Some of the genes with a potential role in CLL biology include E-cadherin [24], CDKN2B [30], BAX [27], and c-Jun [28]. E-cadherin and CDKN2B are known tumor suppressor genes that are hypo-acetylated or silenced in CLL, and the array data shows that this is reversed with HDACi. Similarly, Bax is a pro-apoptotic member of the BCL2 family that is hypo-acetylated in CLL specimens (Figure 3) and re-expressed with HDACi treatment. Gene promoters with an increase histone acetylation 


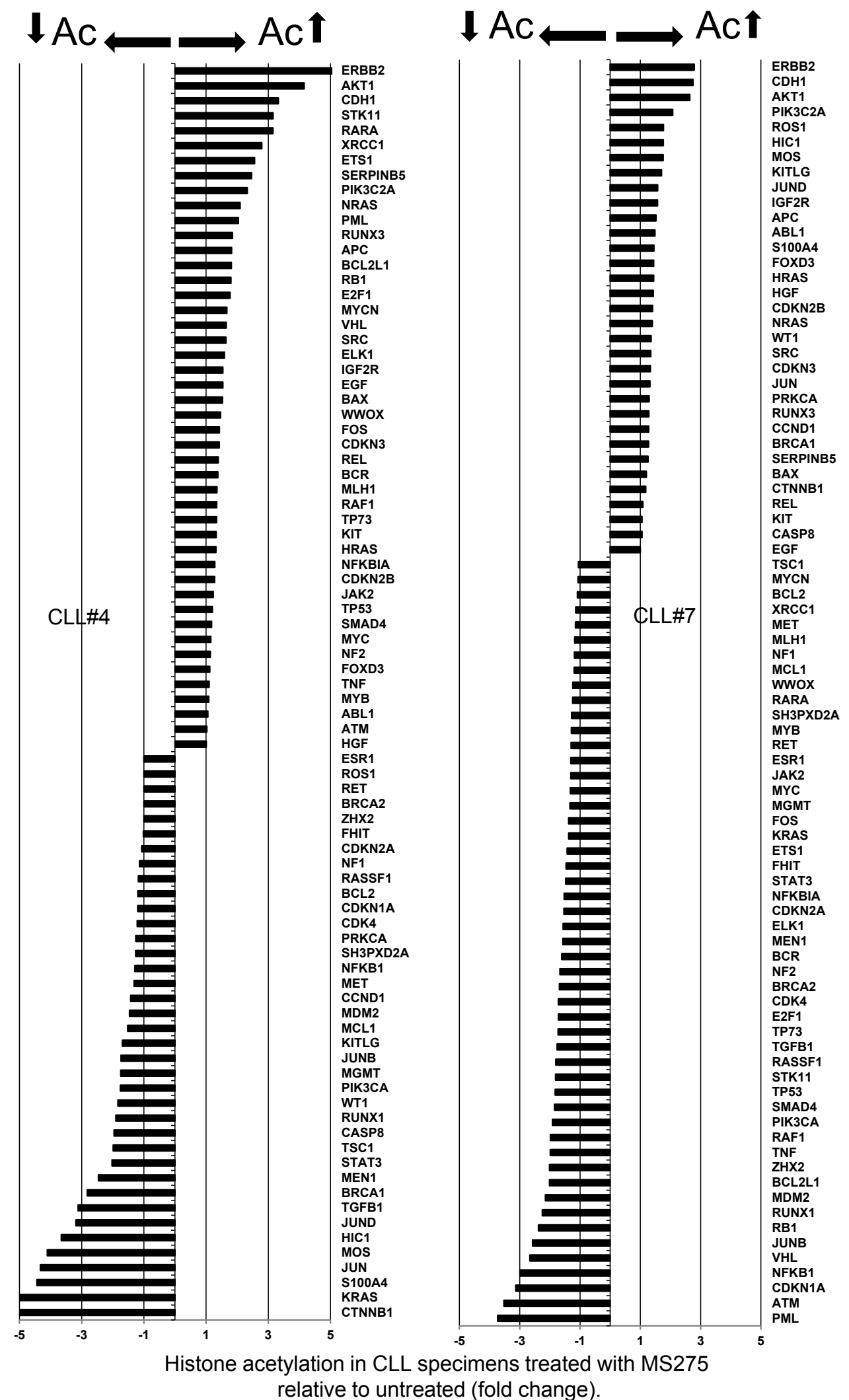

Figure 3: ChIP qPCR array analysis of $\mathrm{H} 4$ acetylation status of promoter regions of genes on the array with HDACi exposure. CLL\#4, CLL\#7 specimens were treated HDACi MS275 and the enrichment index of the treated sample was divided by the enrichment index of the non-treated sample. Gene promoters who are hypo-acetylated with HDACi exposure are plotted towards the left of midline (negative numbers) and the gene promoters who increase histone acetylation are plotted towards the right of the midline (positive numbers). 
A

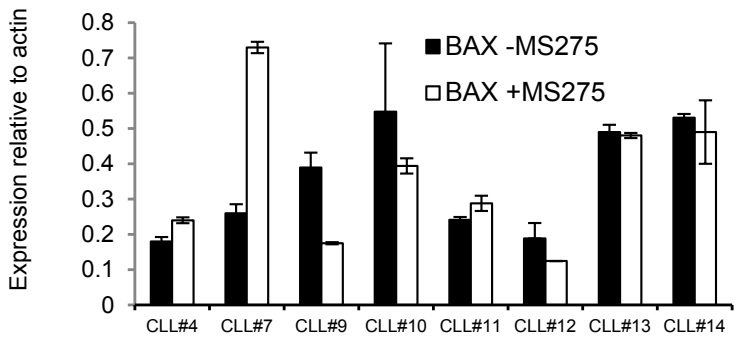

C

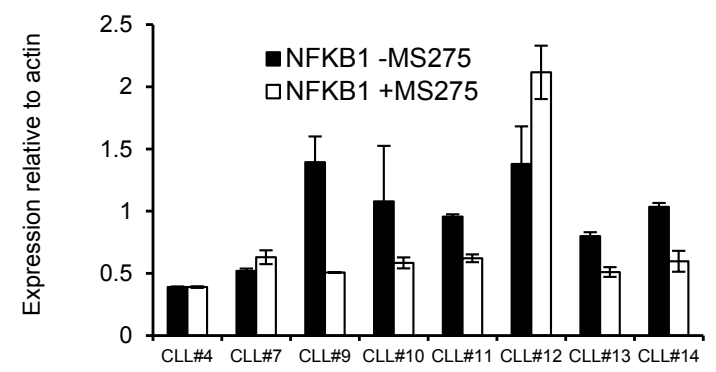

B

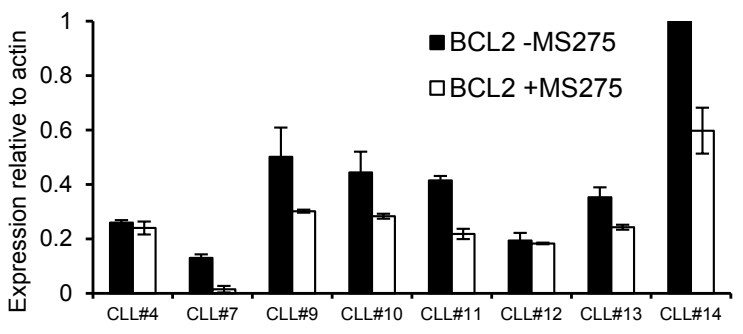

D

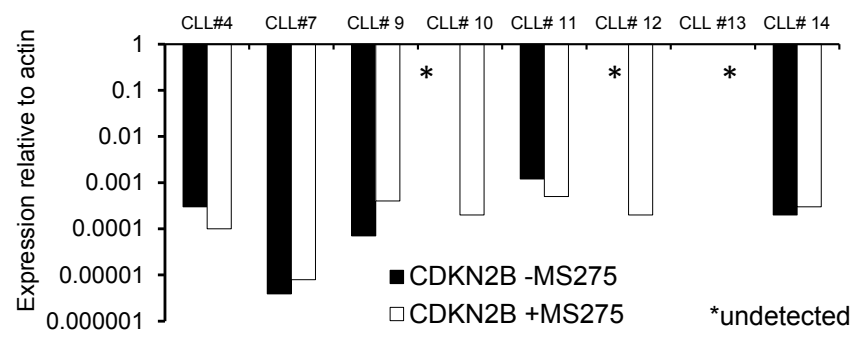

Figure 4: The effects of MS275 treatment on the expression of selected genes. (A-E) Real-time PCR analysis of untreated and MS275 treated primary CLL specimens for BAX, BCL2, NFKB, and CDKN2B RNA expression. All data is adjusted to actin (mean and SD).

with HDACi include genes that are tumor suppressors, inhibitors of growth or mediators of apoptosis indicating that inhibition of their expression with epigenetic modifications potentially contributes to leukemogenesis as reversal of the changes results in apoptosis.

The other set of gene promoters (Figure 3 ) on the contrary, undergo paradoxical histone hypo-acetylation when treated with a drug that blocks histone deactylases. The genes in this group are on the left side of the bar diagram and include genes such as NFKB1, TGFB1, CDK4, MCL1, BCL2, cyclin D1. Functionally these genes are different from the previously described set of genes with hypo-acetylated promoters as they are pro-growth and anti-apoptotic genes. There is thus both histone hypo- and hyper-acetylation with HDACi exposure in gene promoters. The findings indicate that as compared to the non-HDACi treated CLL specimens, the HDACi exposure alters the promoter histone acetylation pattern in a gene dependent manner.

\section{Validation by RT-PCR on additional CLL specimens}

To determine whether histone acetylation ChIP array data with HDACi correlates with changes in RNA expression, a real time PCR analysis was performed on selected genes. The fold change in the RNA expression data in the HDACi treated versus the non-treated CLL specimens was compared to the change in promoter acetylation status (data adjusted to actin). BAX is a pro-apoptotic gene that was silenced in a number of CLL specimens (Figures 1 and 2). In CLL\#4 and \#7 the promoter acetylation status and RNA expression analysis show concordance as the expression of BAX [27] RNA is increased (Figure $4 \mathrm{~A})$ and the promoter is hyper-acetylated with HDACi exposure. When additional CLL specimens were analyzed an increase in BAX expression was only observed in one of six CLL specimens, and the overall data was not statistically significant. On the other hand, BCL2 RNA expression (Figure 4) does correspond to the hypo-acetylation status of its promoter after HDACi exposure as its expression decreased in the six additional CLL specimens that were analyzed ( $\mathrm{p}$ value $=0.2$ ). NFKB1 [34] expression in HDACi treated CLL specimens was detected to be lower than the untreated CLL specimens (in five of eight CLL specimens tested, $\mathrm{p}=0.4)$ that correlates with the hypo-acetylation observed on the ChIP array (Figure 4). In the ChIP analysis of HDACi treated CLL specimens the CDKN2B gene promoter was identified as hypo-acetylated and in the HDACi treated CLL specimens, a higher CDKN2B expression by real time PCR was detected in five of seven CLL specimens indicating the reversal of the epigenetic modification (Figure 4). In two CLL specimens, no CDKN2B expression gene was detected in the untreated leukemic cells while this gene was expressed with HDACi exposure. The data indicates that there is a correlation between the findings on a ChIP-PCR array and RNA expression when cells are treated with HDACi.

\section{Discussion}

This study utilizes a ChIP PCR approach to identify epigenetically altered genes in primary CLL specimens. The study revealed that as compared to PBMC cells, the CLL cells had higher histone H4 hypoacetylated gene promoters. These findings were also validated the ChIP array findings by showing a correlation between RNA expression and acetylation status of the gene promoters along with identification of the novel epigenetically silenced genes that are relevant to the CLL biology. The approach is also valuable in identifying the changes in gene promoters who are altered by HDAC inhibitors as an increase of the acetylated histones in the region of promoters can be easily determined. The data in these experiments indicates that histone acetylation with HDACi is limited to a subset of promoters only and is not global.

Silencing could be due to both epigenetic modifications and other regulatory mechanisms as the histone acetylation status of the promoter reflects the overall transcriptional state of the gene. In this study only the histone epigenetic modifications were analyzed. However, the gene silencing can also be due to methylation and other epigenetic alterations. There was also a correlation between the acetylation status of the promoter and gene expression by RT-PCR for the genes (BAX, BCL2, CDK4 and CDKN2B) that were tested with this approach. Some of the genes tested e.g. BCL2 was only hyper-acetylated in the ChIP array in one of the two CLL specimens. One reason for the lack of 
correlation between acetylation status and gene expression could be that PBMC cells were used as a control specimen for the ChIP array assay as it is technically difficult to obtain purified peripheral blood $\mathrm{B}$ cells in sufficient numbers and is a drawback of this study. Another reason for lack of correlation is that the real time PCR expression data was normalized to purified CD19+ B cells.

BAX and BCL2 expression has been studied by a number of groups, and their ratios at the protein level are known to have a prognostic significance [27]. BAX is a pro-apoptotic gene that is silenced in CLL specimens while BCL2 an anti-apoptotic gene that is upregulated. CDK4 is a protein kinase that is active in G1-S transition and is inhibited by cyclin-dependent kinase inhibitors encoded by CDKN2A, and $2 \mathrm{~B}$ that are known tumor suppressor genes. In the array, the CDKN2B gene is found to have a hypo-acetylated promoter in both the CLL specimens while the CDK4 promoter was one of the few promoters which were hyper-acetylated in CLL specimens as compared to PBMC cells implying increased expression of this kinase in CLL specimens. These findings were confirmed by the expression analysis in additional CLL specimens that confirmed increased RNA expression of CDK4 and loss of expression of CDKN2B. In some cases, the lack of expression of CDKN2B could be secondary to genomic losses of this gene.

The mechanism of action of HDACi is cell type dependent. In CLL specimens, prior reports have indicated an alteration in the genes of the apoptotic pathway. For example, it was reported that there is an increase of pro-apoptotic genes such as BIM and PUMA with SAHA and Kendine 92 HDACi [33]. Treatment with these HDACi also resulted in a downregulation of BCL2 and XIAP, two anti-apoptotic genes. The effect of HDACi on CLL specimens was also studied by an expression microarray to determine genes that alter expression [35]. In this study BCL2, XIAP, c-FLIP was downregulated and CDKN2C, Cyclin E1 were upregulated with HDACi exposure. It is evident from this study and previous reports that not all gene promoters undergo histone hyper-acetylation when treated with HDAC inhibitors. This was confirmed in additional CLL specimens with BCL2 and NFKB1 promoters that also showed a decrease in RNA transcripts with HDACi exposure. Both these genes are known to be active in pro-growth and survival pathways. As CLL specimens undergo apoptosis with HDACi treatment, the data indicates that other regulatory mechanisms play a role, and the expression of pro-survival and anti-apoptotic genes are inhibited in this process. The inhibition of histone deacetylases is no longer able to increase histone acetylation at certain gene promoters. One explanation is that exposure of HDACi initiates cells on an apoptotic pathway which in turn inhibits the expression of certain anti-apoptotic genes such as BCL2 and NFKB1 that results in hypoacetylation of promoters.

Newer techniques aim at a complete genomic profiling of all the histone modifications in a cell [18-20] by a high throughput sequencing approach. The data generated by these techniques is vast and difficult to analyze. The algorithms for data analysis are still in the development stage, and the process requires high level of bioinformatics expertise. The ChIP array described here is a rapid test that can be easily performed and analyzes a number of gene promoters for the histone modifications. A number of epigenetically modified genes identified by the ChIP array were also confirmed by qRT-PCR assays indicating that this assay can be used for screening and investigating the mechanism of action of HDAC inhibitors. By selecting the antibody used for immunoprecipitation a number of specific histone modifications can be analyzed such as methylation and acetylation on specific histone residues. This is relevant as specific histone modifications such as $\mathrm{H} 3 \mathrm{~K} 9 \mathrm{me} 2 / 3$ and $\mathrm{H} 3 \mathrm{~K} 27 \mathrm{me} 3$ histones are associated with gene silencing while active transcription units are associated with lack of $\mathrm{H} 3 \mathrm{~K} 27 \mathrm{me} 3$ and an increase in $\mathrm{H} 3 \mathrm{~K} 4 \mathrm{me} 2, \mathrm{H} 3 \mathrm{~K} 9 \mathrm{ac}$ and $\mathrm{H} 3 \mathrm{~K} 4 \mathrm{ac}$ [36]. The ChIP promoter array can be further modified to include promoters of genes that are of interest in the CLL model and can be developed as a prognostic marker array to identify epigenetic alterations and correlation with disease outcome.

\section{Acknowledgement}

This work was supported by a grant from Flight Attendant Medical Research Institute Fund (FAMRI) and Veterans Administration Merit Review Grant to SS.

\section{References}

1. Ellis L Atadja PW, Johnstone RW (2009) Epigenetics in cancer: targeting chromatin modifications. Mol Cancer Ther 8: 1409-1420.

2. Füllgrabe J Kavanagh E, Joseph B (2011) Histone onco-modifications Oncogene 30: 3391-3403.

3. Neff T Armstrong SA (2009) Chromatin maps, histone modifications and leukemia. Leukemia 23: 1243-1251.

4. Döhner H Stilgenbauer S, Döhner K, Bentz M, Lichter P (1999) Chromosome aberrations in B-cell chronic lymphocytic leukemia: reassessment based on molecular cytogenetic analysis. J Mol Med (Berl) 77: 266-281.

5. Cahill N Rosenquist R (2013) Uncovering the DNA methylome in chronic lymphocytic leukemia. Epigenetics 8: 138-148.

6. Kanduri M Cahill N, Göransson H, Enström C, Ryan F, et al. (2010) Differential genome-wide array-based methylation profiles in prognostic subsets of chronic lymphocytic leukemia. Blood 115: 296-305.

7. Plass C, Byrd JC, Raval A, Tanner SM, De la Chapelle A (2007) Molecular profiling of chronic lymphocytic leukaemia: genetics meets epigenetics to identify predisposing genes. Br J Haematol 139: 744-752.

8. Chim CS Pang R, Liang R (2008) Epigenetic dysregulation of the Wnt signalling pathway in chronic lymphocytic leukaemia. J Clin Pathol 61: 1214-1219.

9. Liu TH Raval A, Chen SS, Matkovic JJ, Byrd JC, et al. (2006) CpG island methylation and expression of the secreted frizzled-related protein gene family in chronic lymphocytic leukemia. Cancer Res 66: 653-658.

10. Stilgenbauer S (2005) ZAP-70 methylation and expression status in chronic lymphocytic leukemia. Haematologica 90: 1012.

11. Corcoran M Parker A, Orchard J, Davis Z, Wirtz M, et al. (2005) ZAP-70 methylation status is associated with ZAP-70 expression status in chronic lymphocytic leukemia. Haematologica 90: 1078-1088.

12. Chim CS Fung TK, Wong KF, Lau JS, Liang R (2006) Frequent DAP kinase but not p14 or Apaf-1 hypermethylation in B-cell chronic lymphocytic leukemia. J Hum Genet 51: 832-838.

13. Baer C Claus R, Frenzel LP, Zucknick M, Park YJ, et al. (2012) Extensive promoter DNA hypermethylation and hypomethylation is associated with aberrant microRNA expression in chronic lymphocytic leukemia. Cancer Res 72: $3775-3785$

14. Berger SL Felsenfeld G (2001) Chromatin goes global. Mol Cell 8: 263-268.

15. Lane AA Chabner BA (2009) Histone deacetylase inhibitors in cancer therapy. $\mathrm{J}$ Clin Oncol 27: 5459-5468.

16. Clayton AL Hazzalin CA, Mahadevan LC (2006) Enhanced histone acetylation and transcription: a dynamic perspective. Mol Cell 23: 289-296.

17. Bokelmann I Mahlknecht U (2008) Valproic acid sensitizes chronic lymphocytic leukemia cells to apoptosis and restores the balance between pro- and antiapoptotic proteins. Mol Med 14: 20-27.

18. Barski A Cuddapah S, Cui K, Roh TY, Schones DE, et al. (2007) High-resolution profiling of histone methylations in the human genome. Cell 129: 823-837.

19. Rahmatpanah FB Carstens S, Hooshmand SI, Welsh EC, Sjahputera O, et al. (2009) Large-scale analysis of DNA methylation in chronic lymphocytic leukemia. Epigenomics 1: 39-61.

20. Tong WG, Wierda WG, Lin E, Kuang SQ, Bekele BN, et al. (2010) Genomewide DNA methylation profiling of chronic lymphocytic leukemia allows 
Citation: Jordaan G, Liao W, Coriaty N, Sharma S (2014) Identification of Histone Epigenetic Modifications with Chromatin Immunoprecipitation PCR Array in Chronic Lymphocytic Leukemia Specimens. J Cancer Sci Ther 6: 325-332. doi:10.4172/1948-5956.1000290

identification of epigenetically repressed molecular pathways with clinical impact. Epigenetics 5: 499-508

21. Pfaffl MW (2001) A new mathematical model for relative quantification in realtime RT-PCR. Nucleic Acids Res 29: e45.

22. Zhang W, Kater AP, Widhopf GF, Chuang HY, Enzler T, et al. (2010) B-cell activating factor and v-Myc myelocytomatosis viral oncogene homolog (c-Myc) influence progression of chronic lymphocytic leukemia. Proc Natl Acad Sci U S A 107: 8956-8960.

23. Decker T Schneller F, Hipp S, Miething C, Jahn T, et al. (2002) Cell cycle progression of chronic lymphocytic leukemia cells is controlled by cyclin D2, cyclin D3, cyclin-dependent kinase (cdk) 4 and the cdk inhibitor p27. Leukemia 16: $327-334$

24. Jordaan G Liao W, Sharma S (2013) E-cadherin gene re-expression in chronic lymphocytic leukemia cells by HDAC inhibitors. BMC Cancer 13: 88.

25. Hanada M Delia D, Aiello A, Stadtmauer E, Reed JC (1993) bcl-2 gene hypomethylation and high-level expression in B-cell chronic lymphocytic leukemia. Blood 82: 1820-1828.

26. Gora-Tybor J, Blonski JZ, Robak T. (2003) Circulating proangiogenic cytokines and angiogenesis inhibitor endostatin in untreated patients with chronic lymphocytic leukemia. Mediators Inflamm 12: 167-171.

27. Saxena A Viswanathan S, Moshynska O, Tandon P, Sankaran K, et al. (2004) $\mathrm{Mcl}-1$ and $\mathrm{Bcl}-2 / \mathrm{Bax}$ ratio are associated with treatment response but not with Rai stage in B-cell chronic lymphocytic leukemia. Am J Hematol 75: 22-33.

28. Jabbar SA Hoffbrand AV, Wickremasinghe RG (1995) Defects in signal transduction pathways in chronic B lymphocytic leukemia cells. Leuk Lymphoma 18: 163-170.
29. Pekarsky Y Palamarchuk A, Maximov V, Efanov A, Nazaryan N, et al. (2008) Tcl1 functions as a transcriptional regulator and is directly involved in the pathogenesis of CLL. Proc Natl Acad Sci U S A 105: 19643-19648.

30. Haidar MA Cao XB, Manshouri T, Chan LL, Glassman A, et al. (1995) p16INK4A and p15INK4B gene deletions in primary leukemias. Blood 86: 311-315

31. Chim CS, Fung TK, Wong KF, Lau JS, Law M, et al. (2006) Methylation of INK4 and CIP/KIP families of cyclin-dependent kinase inhibitor in chronic lymphocytic leukaemia in Chinese patients. J Clin Pathol 59: 921-926.

32. El-Khoury V Moussay E, Janji B, Palissot V, Aouali N, et al. (2010) The histone deacetylase inhibitor MGCD0103 induces apoptosis in B-cell chronic lymphocytic leukemia cells through a mitochondria-mediated caspase activation cascade. Mol Cancer Ther 9: 1349-1360.

33. Perez-Perarnau A, Coll-Mulet L, Rubio-Patino C, Iglesias-Serret D, Cosialls AM, et al. (2011) Analysis of apoptosis regulatory genes altered by histone deacetylase inhibitors in chronic lymphocytic leukemia cells. Epigenetics 6 1228-1235.

34. Herishanu Y, Perez-Galan P, Liu D, Biancotto A, Pittaluga S, et al. (2011) The lymph node microenvironment promotes B-cell receptor signaling, NF-kappaB activation, and tumor proliferation in chronic lymphocytic leukemia. Blood 117: 563-574.

35. Stamatopoulos B Meuleman N, De Bruyn C, Mineur P, Martiat P, et al. (2009) Antileukemic activity of valproic acid in chronic lymphocytic leukemia B cells defined by microarray analysis. Leukemia 23: 2281-2289.

36. Lee BM Mahadevan LC (2009) Stability of histone modifications across mammalian genomes: implications for 'epigenetic' marking. J Cell Biochem 108: $22-34$. 